\title{
Efficacy of brief motivational interviewing on smoking cessation at tuberculosis clinics in Tshwane, South Africa: a randomized controlled trial
}

\author{
Goedele M. C. Louwagie', Kolawole S. Okuyemi ${ }^{2}$ \& Olalekan A. Ayo-Yusuf ${ }^{1,3}$ \\ School of Health Systems and Public Health, Faculty of Health Sciences, University of Pretoria, South Africa,' Department of Family Medicine and Community \\ Health, University of Minnesota, Minneapolis, MN, USA² and Dean/Director's Office, School of Oral Health Sciences, Faculty of Health Sciences, University of \\ Limpopo, Medunsa Campus, South Africa ${ }^{3}$
}

\begin{abstract}
Background and Aims Tuberculosis (TB) patients who smoke risk adverse TB outcomes and other long-term health effects of smoking. This study aimed to determine the efficacy of brief motivational interviewing by lay health-care workers (LHCWs) in assisting TB patients to quit smoking. Design Multi-centre two-group parallel individual randomized controlled trial. Setting Six primary care tuberculosis clinics in a South African township. Participants Newly diagnosed adult TB patients identified as current smokers were randomized to brief motivational interviewing by a LHCW (intervention group, $n=205$ ) or brief smoking cessation advice from a TB nurse (control group, $n=204$ ). Measurements The primary outcome was self-reported sustained 6-month smoking abstinence. Exhaled carbon monoxide (CO) testing was offered to about half the participants. Secondary outcomes were sustained abstinence at 3 months; 7-day point prevalence abstinence at 1, 3 and 6 months; and quit attempts. Allocation was concealed. Primary analysis relied on intention to treat. Multi-level analysis accounted for site heterogeneity of effect. Findings Self-reported 6-month sustained abstinence was $21.5 \%$ for the intervention group versus $9.3 \%$ for the control group [relative risk $(\mathrm{RR})=2.29,95 \%$ confidence interval $(\mathrm{CI})=1.34,3.92$ ]. Biochemically verified 6-month sustained abstinence was also higher in the intervention group (RR 2.21, 95\% CI = 1.08, 4.51) for the 166 participants who were offered carbon monoxide testing. Self-reported 3-month sustained abstinence was $25.4 \%$ for the intervention group and $12.8 \%$ for the control group $(\mathrm{RR}=1.98,95 \% \mathrm{CI}=1.24,3.18)$. Conclusions Motivational interviewing by lay counsellors to promote smoking cessation in tuberculosis patients in South Africa approximately doubled sustained smoking abstinence for at least 6 months compared with brief advice alone.
\end{abstract}

Keywords HIV, lay health-care worker, randomized controlled trial, tobacco, tobacco cessation, tuberculosis.

Correspondence to: Goedele M. C. Louwagie, Faculty of Health Sciences, School of Health Systems and Public Health, Private Bag X 323, Pretoria, 0001 SA, South Africa. E-mail: Goedele.louwagie@up.ac.za

\section{INTRODUCTION}

South Africa has the third highest number of incident tuberculosis (TB) cases in the world [1]. Infection with the human immunodeficiency virus (HIV) is an important driver of the epidemic, with coinfection rates of approximately $60 \%$ [1]. Treatment success rates remain low; death and treatment interruption are the most frequent negative outcomes [1]. The importance of integrating HIV and TB services is now recognized, and progress in integrating these services has been made [1]. Another potentially important driver of the TB epidemic and contributor to poor TB treatment outcomes is active tobacco smoking, which is an established risk factor for TB infection, progression to active TB disease and mortality from TB [2-4]. Smokers also have more severe clinical manifestations and poorer outcomes, aside from dying from TB. In particular, smokers appear to be at increased risk of delayed sputum conversion [5], TB treatment failure [6], TB recurrence [7-9] and possibly drug resistance [10]. Tobacco smoke impairs lung defence mechanisms through structural changes and alterations in cellular and humoural immunity [11-14]. Behavioural characteristics associated with smokers-in particular 
lower drug adherence $[15,16]$ —may also partially explain poorer outcomes for TB patients who smoke tobacco. Tobacco smoking is even more harmful for HIV/TB coinfected patients who are at risk of additional short- and long-term mortality and morbidity due to tobacco smoking and HIV infection [17-21]. As patients with HIV survive longer in the era of highly active antiretroviral therapy (HAART), it becomes increasingly important to prevent these long-term adverse health outcomes related to tobacco smoking.

Smoking cessation is one intervention that can be introduced relatively easily. Both TB and smoking affect the lungs, so being diagnosed with TB provides an ideal learning opportunity [22]. Several studies report promising exploratory and piloting results about integrating tobacco cessation services with TB care [22-26]. In a large cluster-randomized controlled trial in Pakistan, two behavioural support sessions with or without bupropion were highly effective in assisting patients with suspected TB with quitting [27]. To our knowledge, no tobacco cessation trials have been undertaken in a population of TB patients with high HIV coinfection rates, where effectiveness may differ, because dually infected TB patients may be severely ill and/or may have negative outcome expectancies about life, which may function as either a positive or a negative motivator for quitting. The provision of ART care and smoking cessation services, along with TB services, adds another layer of complexity to the feasibility of integrating such services in an already understaffed health system. This study therefore aimed to determine the efficacy of brief motivational interviewing by lay health-care workers (LHCWs) in assisting TB patients with tobacco cessation in a setting with high HIV-TB coinfection rates.

\section{METHODS}

\section{Study setting and participants}

The study took place at the six largest TB clinics in Soshanguve, a large urban township in the City of Tshwane Metropolitan Municipality in South Africa, which were purposively selected for the study because of their large number of TB patients. These primary care facilities offer TB diagnosis and treatment, counselling and testing for HIV and referral to antiretroviral treatment services. LHCWs assist with a variety of administrative and counselling tasks at these clinics. For the purposes of this study, eight of these LHCWs were selected and trained as data collectors and tobacco cessation counsellors and were paid a stipend of about 160 US dollars/month. They all had a minimum of 11 years of schooling and had at least 1 year experience as LHCW. The majority had previously completed one or more formal counselling courses.
All adult patients initiating TB treatment at the six clinics were approached to participate in the study. Participants were excluded if they were under 18 , too ill to participate, unable to understand one of the two languages in which the questionnaire was administered, not currently smoking and had already been on TB treatment for more than 1 month.

The initial planned sample size was 548, based on an assumed $10 \%$ sustained quit rate in controls and $20 \%$ in the intervention group, with $80 \%$ power (two-sided test) and including 20\% attrition. These percentages were based on estimates from another study protocol on smoking cessation in TB patients [28] and on the assumption that TB patients may be particularly motivated to quit $[22,23]$. However, due to slower recruitment of smokers than anticipated for the period of project funding, permission was granted by the institutional ethics committee to review the actual number of smokers needed. A sample size of a minimum of 400 was estimated, based on a more conservative estimate of $5 \%$ cessation in the control group and $15 \%$ in the intervention group, taking into account $20 \%$ attrition.

\section{Trial design and procedures}

This was a multi-centre, two-group, parallel, individual randomized controlled trial (RCT). LHCWs identified current smokers among patients awaiting registration as TB patients at the six clinics, using a baseline screening questionnaire. No validation of self-reported current smoking status was performed at that stage. Current smokers were then allocated by the LHCWs to either the intervention or the control arm by means of sequentially numbered sealed opaque envelopes, thus ensuring allocation concealment. The randomization sequence was generated by an independent epidemiologist who was not otherwise involved in the research project, with a $1: 1$ allocation and random block sizes of $2,4,6,8$ and 10. Participants allocated to the intervention arm received a brief motivational interviewing (MI) session (15-20 minutes) from the LHCW, and were then referred to the TB nurse-who was working in another consultation room-for TB treatment. All participants, whether they belonged to the randomized intervention or control group, received the following short standardized smoking cessation message from the TB nurse: 'Tobacco use is extremely harmful for your health. If you stop smoking now, your TB will heal better and you will have a lower risk of getting TB again in the future. You will also reduce your risk of heart disease and cancer and protect your children against TB. As a professional nurse, I advise you to stop using tobacco in the interests of your health.' All patients also received a 
smoking cessation booklet supplied by the National Council against Smoking of South Africa [29]. It was not possible to blind respondents and LHCWs to the intervention received, because there was only one LHCW per site at four of the six sites. However, TB nurses were blinded to the allocation arm.

Follow-up questionnaires were administered by the LHCWs at participants' routine 1-, 3- and 6-month TB treatment visits. Participants who missed the planned visit were reminded by telephone of the follow-up interview. Participants unable to come back to the clinic in person were interviewed by telephone.

The TB nurse in charge of each clinic underwent 1-day training on the project and in delivering a brief tobacco cessation message. LHCWs received in-depth training on administering questionnaires, enrolment and randomization procedures and a 3-day MI training. Weekly to 2-weekly supervisory site visits were undertaken by the research coordinators to each site for the duration of the project. The baseline leg of the study was piloted at each clinic in a staggered fashion over the course of 6 weeks, after which minor changes were made to the questionnaires.

\section{Intervention}

The intervention was based on 'brief MI' [30]. MI is defined as a 'directive, client-centred counselling style for eliciting behaviour change by helping clients to explore and resolve ambivalence' [31]. This method is moderately effective for various clinical conditions, including smoking cessation [32-34]. A short form, commonly referred to as 'brief MI' has been developed for busy clinical settings. For smoking cessation, the simple one-page approach consists of a quick assessment, the patient identifying problems and solutions and the setting of targets [30]. This was the approach used in our study. Messages about risks, when appropriate, were tailored to the relationship between smoking and TB. LHCWs helped patients who were already highly motivated to quit and were highly confident about their ability to quit with a quit plan [35]. LHCWs received 3 days' in-depth training in tobacco cessation and brief MI for tobacco cessation from an experienced brief MI counsellor and trainer. Training involved video-taped role-play with feedback sessions that ensured that counsellors understood the principles and spirit of MI, and were able to apply the primary skills of using open-ended questions, affirmations, reflective listening and summaries consistently during patient encounters. No pre-post training test was administered immediately after the training. However, as a start-up work-shop alone may be insufficient [36,37], on-site follow-up practical sessions were organized approximately every 4 months with non-videotaped role plays and informal reinforcement of knowledge and skills.

\section{Outcome measures}

Outcome measurements were according to the Russell Standard criteria [38] with some variation-such as not allowing for lapses and the fact that not all participants had a biochemically verified outcome measure. The primary outcome was self-reported 6-month sustained abstinence. Respondents were asked: 'Let's now look back at the period from our very first interview (about 6 months ago) and now. Ignoring the first 2 weeks after the interview, have you smoked any cigarette or smoked any other tobacco product — even a part—since the very first interview?' A similar question was asked at 3-month follow-up. These questions were followed by questions on permissible lapses as defined by Hughes et al. [39]. However, in the actual analysis, a more stringent criterion of "no smoking at all after the initial window period' was used, as participants often appeared to misunderstand the complex questions relating to permissible lapses.

Participants with missing results at 1 or 3 months could still be regarded as a success at 6 months provided they had not smoked at all for the past 6 months [38]. However, if participants made conflicting statementsfor example, self-reporting of smoking at 3 months while declaring sustained abstinence at 6 months-they were classified as continued smokers.

Secondary outcomes were: sustained 3-month abstinence, 7-day point-prevalence abstinence (PPA) at 1, 3 and 6 months after the intervention, and quit attempts (defined as not smoking for 24 hours or more with the intention to quit). At the 1-month follow-up, we also enquired about the receipt of brief cessation advice and the smoking cessation booklet from the TB nurse at the enrolment visit.

Self-reported smoking abstinence was biochemically verified with the piCO ${ }^{+}$Smokerlyzer carbon monoxide (CO) monitor (Bedfont Scientific Ltd, Maidstone, UK) using the standard recommended cut-off value, whereby a reading of 10 parts per million (p.p.m.) or more signifies smoking [38]. CO was selected as the preferred monitoring device because of its ease of use and because it excludes users of smokeless tobacco [38,40,41]. Due to financial constraints, the only three available exhaled CO monitors were rotated to half the clinics, and changed over to the other half every second month. Therefore, about half of all respondents were offered testing at follow-up visits. However, as patients did not know whether the monitor was allocated to their clinics at specific time-points, this approach introduced a bogus pipeline procedure, thus increasing the likelihood of truthful answers. 
For each follow-up period, 4 weeks post-dating the actual follow-up date was allowed [38]. Moreover, the following pre-dating periods were permitted: 1 week for the 1-month follow-up, 3 weeks for the 3-month follow-up and 4 weeks for the 6-month follow-up.

\section{Baseline measures}

Questionnaires were translated into the most commonly used local language and back-translated to English to ensure accuracy of translation, and implemented in this translation and in English. TB- and HIV-related information was obtained from the participants' standardized individual TB records. The questions on tobacco use were adapted from the Global Adult Tobacco Survey questionnaire [42]. We used the Heaviness of Smoking Index (HSI) ('how soon after you wake up do you smoke your first cigarette' and 'how many cigarettes do you smoke per day?') as an indicator of tobacco dependence [43].

We collected information on demographic, socioeconomic and psychosocial indicators which may influence smoking cessation rates: recent depressive symptoms [44], perceived stress [45], social support [46], illicit drug use and an alcohol problem [47]. We also enquired about smoking-related characteristics selfefficacy [48], confidence and motivation to quit and stage of change [49].

\section{Ethical considerations}

Informed consent was obtained from all participants, for the smoking baseline screening questionnaire and participation in the trial for participants identified as current smokers. This included information about the nature of the study, the procedures involved, potential benefits and harms, the right to withdraw at any time, alternative treatment, confidentiality and compensation (lunch parcel to the value of two US dollars).

The trial was registered in the South African (DOH-27-0811-3539) and the Pan African (PACTR 201311000695277) Clinical Trials Register and approved by the Ethics Committee of the University of Pretoria (Protocol 116/2011). Funders had no influence on the data collection, analysis or reporting.

\section{Data management and analysis}

All data were double-entered in Microsoft Excel and analysed with Stata, version 12 [50].

Relative risks (RR) for primary and secondary outcomes were estimated using multi-level Poisson regression analysis to account for site heterogeneity of effect. We repeated this analysis adjusted for the following baseline variables, as pre-specified in the study protocol: HSI, age, duration of smoking and alcohol problem. Absolute risk differences were also calculated. To determine biochemically verified smoking abstinence, two types of analysis were performed, one limited to the subgroup of study participants who were offered testing, the other including all study participants. Participants who refused testing were counted as continued smokers.

Primary analysis was by intention-to-treat (ITT): patients lost to follow-up or who came for follow-up outside the window period were assumed to have continued smoking. Results were also analysed as per protocol, whereby non-eligible patients and patients lost to follow-up were excluded from the analysis.

\section{RESULTS}

\section{Participant follow-up and trial execution}

Subjects were recruited from 14 September 2011 to 25 April 2013 and were followed-up until the end of October 2013. Of the 2411 patients screened for eligibility, $69 \mathrm{did}$ not consent to the study, 1505 were not current smokers and the remainder were excluded for other reasons, as listed in Fig. 1. This resulted in 205 participants randomized to the intervention and 204 to the control. Follow-up rates were $82.4,80.2$ and $76.5 \%$ at $1-, 3$ - and 6-month follow-up, respectively, and did not differ significantly by arm. A total of 21 participants died during follow-up [10 in the control group (4.9\%), 11 in the MI group (5.4\%), $P=0.83]$.

\section{Baseline characteristics}

Thirty-six, 42, 44, 122, 102 and 63 smokers were enrolled at the six respective TB facilities. The baseline characteristics of the two arms of the trial were similar, with only minor differences between groups (Table 1).

\section{Outcomes}

As there was heterogeneity of effect across sites, all relative risk results were calculated with facility as random effect in a two-level analysis.

\section{Primary outcome}

Self-reported 6-month sustained abstinence was more than twice as high in the intervention group as in the control group [21.5 versus $9.3 \%, \mathrm{RR}=2.29$, 95\% confidence interval $(\mathrm{CI})=1.34,3.92]$, with an absolute difference of $12 \%$. Results were similar when adjusted for prespecified potential predictors $(\mathrm{RR}=2.31,95 \% \mathrm{CI}=1.33$, 4.01).

At 6 months, 165 participants underwent exhaled CO testing (one participant refused testing and was counted as a continued smoker; the others were not offered testing). Biochemically verified quit rates were signifi- 


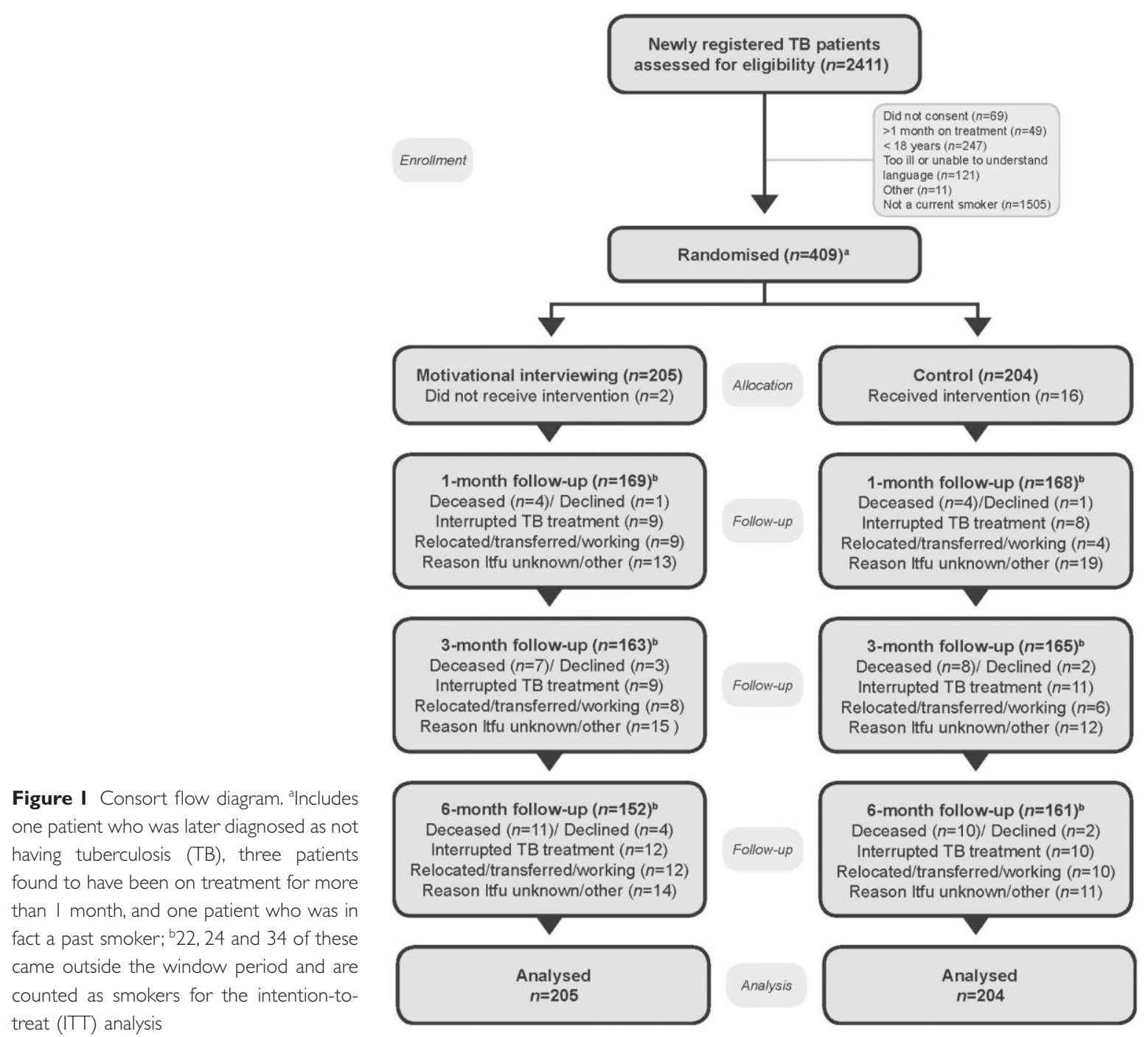

cantly higher in the intervention arm than in the control $\operatorname{arm}(28.9$ versus $13.3 \%$, crude $\mathrm{RR}=2.21,95 \%$ $\mathrm{CI}=1.08,4.51$; adjusted $\mathrm{RR}=2.33,95 \% \mathrm{CI}=1.11$, 4.90) in this subgroup analysis. The association remained significant $(\mathrm{RR}=2.15,95 \% \mathrm{CI}=1.06,4.40)$ when all study participants were included in the denominator (Table 2).

\section{Secondary outcomes}

Three-month sustained abstinence was twice as high in the intervention group as in the control group in both unadjusted $(25.4$ versus $12.8 \%, \quad \mathrm{RR}=1.98, \quad 95 \%$ $\mathrm{CI}=1.24,3.18)$ and adjusted analyses $(\mathrm{RR}=2.04,95 \%$ $\mathrm{CI}=1.24$, 3.35). The biochemically verified 3-month abstinence rates were significantly higher for the intervention group in the adjusted analysis, but not in the crude analysis. Self-reported 7-day PPA was higher in the intervention group at 1- and 3-month follow-up. However, quit attempts did not differ by intervention arm (Table 3).
Similar but slightly stronger associations were found in the per-protocol analyses for both the primary and secondary outcomes (results not presented). Most respondents (87\%) confirmed that the TB nurse enquired about their smoking status at enrolment. In more than two-thirds of cases, the nurse advised the patient to stop smoking in general, without tailoring the advice to TB (68.1\%), about one in 10 patients $(10.3 \%)$ received a TB-tailored smoking cessation message, 11\% were advised to reduce smoking, and the rest did not receive any message (10.6\%). Most smokers received the smoking cessation booklet at baseline (82.4\%), and about three-quarters of these $(74.6 \%)$ reported reading it (Table 4).

\section{DISCUSSION}

The current study showed that brief MI counselling by LHCWs was effective in assisting TB patients to quit tobacco smoking. Both the relative and absolute effects 
Table 1 Baseline characteristics of the participants.

\begin{tabular}{|c|c|c|}
\hline & MI group $(n=205)$ & Control group $(n=204)$ \\
\hline Men, $n / N(\%)$ & $188 / 205(91.7)$ & $180 / 204(88.2)$ \\
\hline Age $[$ mean $(\mathrm{SD})](n=409)$ & $40.3($ SD 10.3) & $42.3(\mathrm{SD} 10.1)$ \\
\hline \multicolumn{3}{|l|}{ Education, $n / N(\%)$} \\
\hline Primary schooling or less & $66 / 200(33.0)$ & $85 / 204(41.7)$ \\
\hline Some high school & $100 / 200(50.0)$ & $94 / 204(46.1)$ \\
\hline Completed high school or higher & $34 / 200(17.0)$ & $25 / 204(12.3)$ \\
\hline \multicolumn{3}{|l|}{ Marital status, $n / N(\%)$} \\
\hline Now married & $52 / 202(25.7)$ & $62 / 204(30.4)$ \\
\hline Divorced/separated/widowed & $16 / 202(7.9)$ & $21 / 204(10.3)$ \\
\hline Never married & $134 / 202(66.3)$ & $121 / 204(59.3)$ \\
\hline Asset score [median (IQR)] $(n=404)$ & $4(3-4)$ & $4(3-4)$ \\
\hline Hungry for $\geq 1$ day/month, $n / N(\%)$ & $30 / 202(14.9)$ & $33 / 203(16.3)$ \\
\hline \multicolumn{3}{|l|}{ Employment category, $n / N(\%)$} \\
\hline Unemployed & $55 / 199(27.6)$ & $60 / 201(29.9)$ \\
\hline Working full- or part-time & $131 / 199(65.8)$ & $130 / 201(64.7)$ \\
\hline Not working ${ }^{\mathrm{a}}$ & $13 / 199(6.5)$ & $11 / 201(5.5)$ \\
\hline \multicolumn{3}{|l|}{ Household earnings, $n / N(\%)$} \\
\hline $\mathrm{ZAR}^{\mathrm{b}} 1-500$ & $74 / 201(36.8)$ & $64 / 202(31.7)$ \\
\hline ZAR 501-2500 & $100 / 201(49.8)$ & $101 / 202(50.0)$ \\
\hline $\mathrm{ZAR}>2500$ & $27 / 201(13.4)$ & $37 / 202(18.3)$ \\
\hline Depressive symptoms (past 2 weeks) & $57 / 181(31.5)$ & $69 / 182(37.9)$ \\
\hline Perceived stress score (range 0-16) [median $(\mathrm{IQR})](n=401)$ & $8(5-10)$ & $8(5-9)$ \\
\hline MOS-social support $^{\mathrm{c}}($ range $1-100)[$ median $(\mathrm{IQR})](n=392)$ & $75(59.2-88.2)$ & $75(56.6-89.5)$ \\
\hline Alcohol problem, ${ }^{\mathrm{d}} n / \mathrm{N}(\%)$ & $109 / 203(53.7)$ & $91 / 199(45.7)$ \\
\hline Illicit drug use, $n / N(\%)$ & 26/199 (13.1) & $33 / 197(16.8)$ \\
\hline First episode of tuberculosis, $n / N(\%)$ & $170 / 198(85.9)$ & $177 / 200(88.5)$ \\
\hline Pulmonary tuberculosis, $n / N(\%)$ & $183 / 200(91.5)$ & $189 / 203(93.1)$ \\
\hline HIV-positive, $n / N(\%)$ & $164 / 187(87.7)$ & $156 / 188(83.0)$ \\
\hline Age started smoking [median (IQR)] $(n=316)$ & $17(15-20)$ & $18(15-20)$ \\
\hline Years of regular smoking [median $(\mathrm{IQR})](n=372)$ & $20.0(14.2-26.8)$ & $20.7(14.4-28.9)$ \\
\hline Daily cigarette consumption ${ }^{\mathrm{e}}$ [median (IQR)] & $8(5-14)$ & $8(5-12)$ \\
\hline Mean $(\mathrm{SD})(n=341)$ & $10.0(\mathrm{SD} 7.1)$ & $9.8(\mathrm{SD} 7.1)$ \\
\hline Heaviness of smoking index $\geq 4, n / N(\%)$ & $37 / 182(20.3)$ & $40 / 188(21.3)$ \\
\hline Quit attempt in past 12 months, $n / N(\%)$ & $104 / 198(52.5)$ & $103 / 200(51.5)$ \\
\hline Motivation score [median (IQR)] (range 1-10) $(n=400)$ & $9(7-10)$ & $9(7-10)$ \\
\hline Confidence score $[$ median $(\mathrm{IQR})]($ range $1-10)(\mathrm{n}=401)$ & $9(6-10)$ & $9(6-10)$ \\
\hline Self-efficacy score [median (IQR)] (range 9-45) $(n=389)$ & $26(18-35)$ & $25(18-36)$ \\
\hline Preparation stage of change, $\mathrm{n} / \mathrm{N}(\%)$ & $106 / 187(56.7)$ & 116/190 (61.1) \\
\hline
\end{tabular}

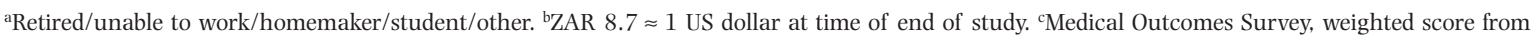
0-100. ${ }^{\mathrm{d}}$ CAGE (Cut-Down, Annoyed, Guilt, Eye-Opener) score $\geq 2$. ${ }^{e}$ Daily smokers only. MI = motivational interviewing; SD = standard deviation; $\mathrm{IQR}=$ interquartile range.

were somewhat higher than results of systematic reviews regarding the effectiveness of $\mathrm{MI}$ in non-TB populations $[33,34]$. The relatively high cessation rates in our study could possibly be partially attributed to very low prior exposure to any type of smoking cessation counselling in our study population [51]. Furthermore, we believe the non-judgemental client-centred approach of MI was crucial to our success rates, in a care context where patients are more often exposed to paternalistic and condescending counselling styles [52]. Lastly, our participants were often very ill with TB and HIV and the link between smoking and TB is easily understood [22].
However, the effect of our intervention was much lower than in the cluster-RCT in patients with suspected TB in Pakistan. The higher quit rates in the Pakistan study could possibly be explained by their more intensive smoking cessation intervention (a behavioural change consultation of 30-40 minutes followed by a second shorter session, with or without buproprion).

Self-reported quit rates were quite high in the control group, and similar to the Pakistan TB study [27]. Some possible explanations are, first, that the brief advice and smoking cessation booklet provided by the TB nurse may have aided participants in quitting; secondly, answering 
Table 2 Primary outcome: 6-month sustained abstinence.

\begin{tabular}{|c|c|c|c|c|c|}
\hline & $\begin{array}{l}\text { Intervention } \\
n(\%)\end{array}$ & $\begin{array}{l}\text { Control } \\
n(\%)\end{array}$ & $\begin{array}{l}\text { Absolute difference } \\
(95 \% \mathrm{CI})\end{array}$ & $\begin{array}{l}\text { Crude RR } \\
(95 \% \text { CI })\end{array}$ & $\begin{array}{l}\text { Adjusted } R R^{\mathrm{b}} \\
(95 \% \mathrm{CI})\end{array}$ \\
\hline $\begin{array}{l}\text { Self-reported 6-month sustained } \\
\text { abstinence }\end{array}$ & $44 / 205$ (21.5) & 19/204 (9.3) & $0.12(0.05 ; 0.19)$ & $2.29(1.34 ; 3.92)$ & $2.31(1.33 ; 4.01)$ \\
\hline $\begin{array}{l}\text { Biochemically verified 6-month } \\
\text { sustained abstinence }{ }^{\mathrm{d}} \\
\text { (participants offered CO testing, } \\
n=166^{\mathrm{e}} \text { ) }\end{array}$ & 24/83 (28.9) & $11 / 83(13.3)$ & $0.16(0.03 ; 0.28)$ & $2.21(1.08 ; 4.51)$ & $2.33(1.11 ; 4.90)$ \\
\hline $\begin{array}{l}\text { Biochemically verified 6-month } \\
\text { sustained abstinence }{ }^{\text {c }} \text { all } \\
\left.\text { participants }{ }^{\mathrm{f}}\right)\end{array}$ & $24 / 205$ (11.7) & $11 / 204(5.4)$ & $0.06(0.01 ; 0.12)$ & $2.15(1.06 ; 4.40)$ & $2.14(1.01 ; 4.51)$ \\
\hline
\end{tabular}

a Univariate multi-level Poisson regression with facility as random effect. ${ }^{b}$ Multi-level Poisson regression with facility as random effect, adjusted for Heaviness of Smoking Index (HSI), age, duration of smoking, alcohol problem, as pre-specified in study protocol ( $n=395$ for entire sample and 161 for the biochemically verified results). ${ }^{c}$ Participants lost to follow-up and those who came outside the 6 -month visit window period were considered to be still smoking. ${ }^{~}$ Self-reported sustained abstinence at 6 months and carbon monoxide $(\mathrm{CO})<10$ parts per million (p.p.m.). ${ }^{\mathrm{e}} 166$ participants were offered CO testing, of whom one refused; the CO-level of the participant who refused was assumed to be $\geq 10$ p.p.m. ${ }^{\mathrm{f}}$ Assumes that all participants not followed-up and those coming outside window period and those not offered CO testing had a CO-level of $\geq 10$ p.p.m. RR $=$ relative risk; CI $=$ confidence interval.

Table 3 Secondary outcomes.

\begin{tabular}{|c|c|c|c|c|c|}
\hline & Intervention & Control & Absolute difference & Crude $R R^{a}$ & Adjusted $R R^{b}$ \\
\hline & $n(\%)$ & $n(\%)$ & $(95 \% C I)$ & $(95 \% C I)^{a}$ & $(95 \% C I)$ \\
\hline \multicolumn{6}{|l|}{ 6-month follow-up } \\
\hline $\begin{array}{l}\text { Self-reported 7-day PPA } \\
\text { 3-month follow-up }\end{array}$ & $92 / 205$ (44.9) & $82 / 204(40.2)$ & $0.05(-0.05 ; 0.14)$ & $1.12(0.83 ; 1.50)$ & $1.09(0.80 ; 1.47)$ \\
\hline $\begin{array}{l}\text { Self-reported sustained 3-month } \\
\text { abstinence }\end{array}$ & $52 / 205(25.4)$ & $26 / 204(12.8)$ & $0.13(0.05 ; 0.20)$ & $1.98(1.24 ; 3.18)$ & $2.04(1.24 ; 3.35)$ \\
\hline $\begin{array}{l}\text { Biochemically verified sustained } \\
\text { 3-month abstinence } \\
\text { (participants offered testing, } \\
n=156^{\mathrm{d}} \text { ) }\end{array}$ & $21 / 83(25.3)$ & $10 / 73(13.7)$ & $0.12(-0.01 ; 0.24)$ & $2.03(0.95 ; 4.33)$ & $2.32(1.02 ; 5.27)$ \\
\hline $\begin{array}{l}\text { Biochemically verified sustained } \\
\text { 3-month abstinence } \\
\quad(\text { all participants, } n=409)^{\mathrm{e}}\end{array}$ & $21 / 205(10.2)$ & $10 / 204(4.9)$ & $0.05(<0.01 ; 0.10)$ & $2.08(0.98 ; 4.42)$ & $2.39(1.05 ; 5.43)$ \\
\hline $\begin{array}{l}\text { Self-reported 7-day } \mathrm{PPA}^{\mathrm{c}} \\
\text { 1-month follow-up }\end{array}$ & $81 / 205(39.5)$ & $56 / 204(27.5)$ & $0.12(0.03 ; 0.21)$ & $1.44(1.02 ; 2.03)$ & $1.44(1.01 ; 2.05)$ \\
\hline Self-reported 7-day $\mathrm{PPA}^{\mathrm{c}}$ & $72 / 205(35.1)$ & $45 / 204(22.1)$ & $0.13(0.04 ; 0.22)$ & $1.59(1.10 ; 2.31)$ & $1.58(1.07 ; 2.34)$ \\
\hline $\begin{array}{l}\text { Biochemically verified 7-day } \\
\text { PPA (participants offered testing, } \\
n=168^{\mathrm{f}} \text { ) }\end{array}$ & $39 / 79(48.4)$ & $25 / 89(28.1)$ & $0.21(0.07 ; 0.36)$ & $1.76(1.06 ; 2.90)$ & $1.74(1.01 ; 3.01)$ \\
\hline $\begin{array}{l}\text { Biochemically verified 7-day } \\
\text { PPA (all participants, } n=409)^{\mathrm{g}}\end{array}$ & $39 / 205(19.0)$ & 25/204 (12.3) & $0.07(>-0.01 ; 0.14)$ & $1.55(0.94 ; 2.56)$ & $1.63(0.95 ; 2.79)$ \\
\hline Successful quit attempt & $139 / 205(67.8)$ & $125 / 204(61.3)$ & $0.07(-0.03 ; 0.16)$ & $1.11(0.87 ; 1.41)$ & $1.09(0.85 ; 1.40)$ \\
\hline
\end{tabular}

${ }^{\mathrm{a}}$ Univariate multi-level Poisson regression with facility as random effect. ${ }^{\mathrm{b}}$ Multi-level Poisson regression with facility as random effect, adjusted for Heaviness of Smoking Index (HSI), age, duration of smoking, alcohol problem, as pre-specified in study protocol ( $n=395$ for entire sample and 151 and 161 for the 3-and 1-month biochemically verified subgroup, respectively). 'Participants lost to follow-up and those who came outside the window period were considered to be still smoking. ${ }^{\mathrm{d}} 156$ participants were offered testing, of whom three refused testing; the carbon monoxide (CO)-level of the three

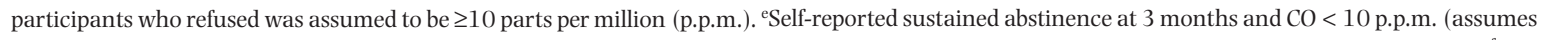
that all participants not followed-up and those coming outside the window period and those not offered $\mathrm{CO}$ testing had a $\mathrm{CO}$ level of $\geq 10$ ). ${ }^{\mathrm{f}} 168$ participants were offered CO testing, of whom six refused testing. ${ }^{g}$ Self-reported 7 -day point prevalence abstinence (PPA) and CO < 10 p.p.m. (assumes that all participants not followed-up and those coming outside the window period and those not offered CO testing had a CO level of $\geq 10$ ). RR $=$ relative risk; $\mathrm{CI}=$ confidence interval. 
Table 4 Delivery of brief cessation message by tuberculosis (TB) nurse; receipt and use of smoking cessation booklet ${ }^{\mathrm{a}}$.

\begin{tabular}{|c|c|c|c|c|}
\hline & All respondents & Intervention & Control & \\
\hline & $n / N(\%)$ & $n / N(\%)$ & $n / N(\%)$ & P-value \\
\hline TB nurse inquired about smoking at baseline & $282 / 324(87.0)$ & $146 / 162(90.1)$ & $136 / 162(84.0)$ & $0.098^{\mathrm{b}}$ \\
\hline \multicolumn{5}{|l|}{ Message given by TB nurse } \\
\hline No message & $30 / 282(10.6)$ & $12 / 146(8.2)$ & $18 / 136(13.2)$ & $0.278^{\mathrm{b}}$ \\
\hline Advised to stop smoking & $192 / 282(68.1)$ & $104 / 146(71.2)$ & $88 / 136(64.7)$ & \\
\hline Advised to reduce smoking & $31 / 282(11.0)$ & $13 / 146(8.9)$ & $18 / 136(13.2)$ & \\
\hline TB-related cessation message & $29 / 282(10.3)$ & 17/146 (11.6) & $12 / 136(8.8)$ & \\
\hline Usefulness score of TB nurse message [median (IQR)] $(n=247)$ & $5(4-5)$ & $5(4-5)$ & $5(4-5)$ & $0.060^{\mathrm{c}}$ \\
\hline Smoking cessation booklet received & $230 / 283(82.4)$ & $121 / 145(83.5)$ & $109 / 134(81.3)$ & $0.644^{\mathrm{b}}$ \\
\hline Read any part of smoking cessation booklet & $160 / 224(74.6)$ & $69 / 105(69.1)$ & $91 / 119(79.1)$ & $0.089^{\mathrm{b}}$ \\
\hline
\end{tabular}

${ }^{a}$ All questions posed at the 1-month follow-up visit; analysis limited to participants followed-up at 1 month. ${ }^{\text {b }} \chi^{2}$ test. 'Wilcoxon's rank-sum test. $\mathrm{IQR}=$ interquartile range.

questions on tobacco use, health beliefs and motivation to quit may have led to reflection about the harmful effects of smoking and may have altered subsequent smoking behaviour; lastly, being ill with TB may, in itself, be sufficient to induce people to stop smoking $[22,24,26]$. Considerable effects in the control group have also been found for another behavioural intervention in South African TB patients [53].

There were 21 reported deaths during the trial. It is extremely difficult to obtain the exact causes of death in this community setting, but it is unlikely that these deaths occurred due to the smoking cessation trial. Because all participants were ill with TB and most were also HIV-positive, the most likely cause of death was related to HIV and/or TB [54] or other causes unrelated to tobacco cessation. Death rates did not differ significantly between arms, and the majority of deaths occurred within 3 months after TB registration.

Our study has some limitations. A relatively high percentage of patients could not be traced for one or more of the follow-up visits; however, the loss to follow-up rate was similar in the intervention and control groups. Furthermore, all patients lost to follow-up were considered smokers in the ITT analysis. MI was offered in only a single brief session by LHCWs. We were unable to monitor intervention fidelity by video-taping actual MI sessions with real patients, as it is considered invasive to patients within the context of standard clinical care. It is therefore possible that LHCWs, although intensively trained, did not deliver MI adequately [55]. However, the outcomes were similar or even better than would have been expected with MI. Follow-up measurement was not blinded, which may have introduced respondent- or interviewer-bias. Over-reporting of self-reported quit rates is thus possible, which may partially explain the high self-reported 7-day PPA obtained at 6-month followup. We attempted to minimize over-reporting by offering potential testing with exhaled $\mathrm{CO}$ monitoring. However, a drawback of $\mathrm{CO}$ monitoring is the short half-life of $\mathrm{CO}$ in smokers-morning readings may thus give misleadingly low results [56]. Furthermore, only half the participants were offered testing.

We did not offer pharmacotherapy to smokers, as smoking cessation medication is expensive and currently not available in public primary care clinics in South Africa. There is also insufficient evidence of its effectiveness in light smokers $[57,58]$. The longest follow-up was 6 months. It may have been advisable to follow-up the patients again at 12 months, even more so because there is evidence that TB patients may relapse to smoking when they feel better as the TB is treated $[22,24,26]$. However, 6 months was chosen because this time-period coincides with the minimum duration of TB treatment. Lastly, the results of the trial may not be generalizable to primary care clinics in different settings, but can probably be extrapolated to similar public health clinics in South Africa.

In conclusion, brief MI delivered by LHCWs was effective in assisting TB patients with smoking cessation under trial conditions in a South African setting. Of particular relevance is that this study demonstrates effectiveness in a population of mainly light smokers. It follows from these results that smoking cessation is possible in TB patients with high HIV coinfection rates in a low- to mediumincome country such as South Africa. The second important finding is that smoking cessation services can be delivered by LHCWs - as demonstrated in only a few other studies [59-61] — thus freeing valuable nurse time for more clinical tasks. This is important in the South African context, because TB patients often receive treatment for both TB and HIV. Although these conditions are usually managed in parallel fashion by different providers [62], TB nurses spend a great deal of time referring patients to and from HIV services. Adding intensive smoking cessation 
counselling to their tasks may lead to poor implementation and be unsustainable. Furthermore, the use of LHCWs fits neatly with the enhanced role of LHCWs as outlined in South Africa's National Health Insurance plans [63]. Based on the above findings, we recommend the careful implementation of brief MI by LHCWs at TB clinics in Tshwane. If successful, the same programme could be rolled out to other parts of South Africa. Adding smoking cessation to TB programmes will not only reduce long-term smoking-related complications, but crucially TB and HIV-related treatment outcomes.

\section{Clinical trial registration}

Pan African Clinical Trials PACTR201311000695277 and South African Clinical Trials DOH-27-0811-3539.

\section{Declaration of interests}

K.O. received Pfizer funding for an FDA-approved research project (unrelated to this project) involving the use of nicotine patch, bupropion and varenicline. O.A.A.-Y. is a sub-awardee of an unrestricted Pfizer Education grant to Mayo Clinic for the Global Bridges Health Alliance project and received an honorarium as a speaker at the 2012 congress of the South African Dental Association for a session on treatment funded by Pfizer.

\section{Acknowledgements}

This project was supported by funding from the KNCV Tuberculosis Foundation (grant 12.402.2/MvdW/ U.10.0696/cal), Global Bridges Health Care Alliance for Tobacco Dependence Treatment and the National Research Foundation of South Africa (grant no. 80843). The funders had no role in the conceptualization of the study, data analysis or reporting of results. The National Council against Smoking of South Africa provided the smoking cessation booklets. We are grateful to the fieldworkers and supervisory staff, in particular Ms K. Kodi, for their enthusiastic and hard work. We also wish to thank the TB nurses at the facilities and the Tshwane District Health management team for their support.

\section{References}

1. World Health Organization. Global Tuberculosis Report 2012. Geneva: WHO; 2012. WHO/HTM/TB/2012.6. Available at: http://apps.who.int/iris/bitstream/10665/75938/1/ 9789241564502_eng.pdf (accessed 10 July 2014) (Archived at http://www.webcitation.org/6QxWwwVPs on 10 July 2014).

2. Bates M. N., Khalakdina A., Pai M., Chang L., Lessa F., Smith K. R. Risk of tuberculosis from exposure to tobacco smoke: a systematic review and meta-analysis. Arch Intern Med 2007; 167: 335-42.
3. Lin H. H., Ezzati M., Murray M. Tobacco smoke, indoor air pollution and tuberculosis: a systematic review and metaanalysis. PLoS Med 2007; 4: e20. doi: 10.1371/ journal.pmed.0040020.

4. Slama K., Chiang C. Y., Enarson D. A., Hassmiller K., Fanning A., Gupta P. et al. Tobacco and tuberculosis: a qualitative systematic review and meta-analysis. Int J Tuberc Lung Dis 2007; 11: 1049-61.

5. Maciel E. L., Brioschi A. P., Peres R. L., Guidoni L. M., Ribeiro F. K., Hadad D. J. et al. Smoking and 2-month culture conversion during anti-tuberculosis treatment. Int J Tuberc Lung Dis 2013; 17: 225-8.

6. Tachfouti N., Nejjari C., Benjelloun M. C., Berraho M., Elfakir S., El Rhazi K. et al. Association between smoking status, other factors and tuberculosis treatment failure in Morocco. Int J Tuberc Lung Dis 2011; 15: 838-43.

7. Thomas A., Gopi P. G., Santha T., Chandrasekaran V., Subramani R., Selvakumar N. et al. Predictors of relapse among pulmonary tuberculosis patients treated in a DOTS programme in South India. Int J Tuberc Lung Dis 2005; 9: 556-61.

8. Jee S. H., Golub J. E., Jo J., Park I. S., Ohrr H., Samet J. M. Smoking and risk of tuberculosis incidence, mortality, and recurrence in South Korean men and women. Am J Epidemiol 2009; 170: 1478-85.

9. d' Arc Lyra Batista J., de Fatima Pessoa Militao de Albuquerque M., de Alencar Ximenes R. A., Rodrigues L. C. Smoking increases the risk of relapse after successful tuberculosis treatment. Int J Epidemiol 2008; 37: 841-51.

10. Ruddy M., Balabanova Y., Graham C., Fedorin I., Malomanova N., Elisarova E. et al. Rates of drug resistance and risk factor analysis in civilian and prison patients with tuberculosis in Samara Region, Russia. Thorax 2005; 60: 130-5.

11. Holt P. G. Immune and inflammatory function in cigarette smokers. Thorax 1987; 42: 241-9.

12. Dye J. A., Adler K. B. Effects of cigarette smoke on epithelial cells of the respiratory tract. Thorax 1994; 49: 825-34.

13. Arcavi L., Benowitz N. L. Cigarette smoking and infection. Arch Intern Med 2004; 164: 2206-16.

14. Feldman C., Anderson R. Cigarette smoking and mechanisms of susceptibility to infections of the respiratory tract and other organ systems. J Infect 2013; 67: 169-84.

15. Chang K. C., Leung C. C., Tam C. M. Risk factors for defaulting from anti-tuberculosis treatment under directly observed treatment in Hong Kong. Int J Tuberc Lung Dis 2004; 8: 1492-8.

16. Naidoo P., Peltzer K., Louw J., Matseke G., McHunu G., Tutshana B. Predictors of tuberculosis (TB) and antiretroviral (ARV) medication non-adherence in public primary care patients in South Africa: a cross sectional study. BMC Public Health 2013; 13: 396. doi: 10.1186/ 1471-2458-13-396.

17. Miguez-Burbano M. J., Ashkin D., Rodriguez A., Duncan R., Pitchenik A., Quintero N. et al. Increased risk of Pneumocystis carinii and community-acquired pneumonia with tobacco use in HIV disease. Int J Infect Dis 2005; 9: 208-17.

18. Crothers K., Griffith T. A., McGinnis K. A., Rodriguez-Barradas M. C., Leaf D. A., Weissman S. et al. The impact of cigarette smoking on mortality, quality of life, and comorbid illness among HIV-positive veterans. J Gen Intern Med 2005; 20: 1142-5. 
19. Marshall M. M., McCormack M. C., Kirk G. D. Effect of cigarette smoking on HIV acquisition, progression, and mortality. AIDS Educ Prev 2009; 21: 28-39.

20. van Zyl Smit R. N., Pai M., Yew W. W., Leung C. C., Zumla A., Bateman E. D. et al. Global lung health: the colliding epidemics of tuberculosis, tobacco smoking, HIV and COPD. Eur Respir J 2010; 35: 27-33.

21. Helleberg M., Afzal S., Kronborg G., Larsen C. S., Pedersen G., Pedersen C. et al. Mortality attributable to smoking among HIV-1-infected individuals: a nationwide, population-based cohort study. Clin Infect Dis 2013; 56: 727-34.

22. Shin S. S., Xiao D., Cao M., Wang C., Li Q., Chai W. X. et al. Patient and doctor perspectives on incorporating smoking cessation into tuberculosis care in Beijing, China. Int $J$ Tuberc Lung Dis 2012; 16: 126-31.

23. El Sony A., Slama K., Salieh M., Elhaj H., Adam K., Hassan A. et al. Feasibility of brief tobacco cessation advice for tuberculosis patients: a study from Sudan. Int J Tuberc Lung Dis 2007; 11: 150-5.

24. Pradeepkumar A. S., Thankappan K. R., Nichter M. Smoking among tuberculosis patients in Kerala, India: proactive cessation efforts are urgently needed. Int J Tuberc Lung Dis 2008; 12: 1139-45.

25. Sereno A. B., Soares E. C., Lapa E Silva J. R., Napoles A. M., Bialous S. A., Costa E Silva V. L. et al. Feasibility study of a smoking cessation intervention in Directly Observed Therapy Short-Course tuberculosis treatment clinics in Rio de Janeiro, Brazil. Rev Panam Salud Publica 2012; 32: 4516.

26. Ng N., Padmawati R. S., Prabandari Y. S., Nichter M. Smoking behavior among former tuberculosis patients in Indonesia: intervention is needed. Int $J$ Tuberc Lung Dis 2008; 12: 567-72.

27. Siddiqi K., Khan A., Ahmad M., Dogar O., Kanaan M., Newell J. N. et al. Action to stop smoking in suspected tuberculosis (ASSIST) in Pakistan: a cluster randomized, controlled trial. Ann Intern Med 2013; 158: 667-75.

28. Siddiqi K., Khan A., Ahmad M., Rehman S. U. An intervention to stop smoking among patients suspected of TB-evaluation of an integrated approach. BMC Public Health 2010; 10: 160. doi: 10.1186/1471-2458-10-160.

29. National Council Against Smoking. Make a Fresh Start. Your personal guide to stop smoking. Available at: http:// againstsmoking.co.za/images/makeafreshstart.pdf (accessed 2 May 2014) (Archived at http://www .webcitation.org/6PGbVFrtQ on 2 May 2014).

30. Rollnick S., Butler C. C., Stott N. Helping smokers make decisions: the enhancement of brief intervention for general medical practice. Patient Educ Couns 1997; 31: 191-203.

31. Rollnick S., Miller W. R. What is motivational interviewing? Behav Cogn Psychother 1995; 23: 325-34.

32. Rubak S., Sandbaek A., Lauritzen T., Christensen B. Motivational interviewing: a systematic review and meta-analysis. Br J Gen Pract 2005; 55: 305-12.

33. Lai D. T., Cahill K., Qin Y., Tang J. L. Motivational interviewing for smoking cessation. Cochrane Database Syst Rev 2010; 1: CD006936. doi: 10.1002/14651858.CD006936.pub2.

34. Heckman C. J., Egleston B. L., Hofmann M. T. Efficacy of motivational interviewing for smoking cessation: a systematic review and meta-analysis. Tob Control 2010; 19: 410-6.

35. Fiore M. C., Jaén C. R., Baker T. B., Bailey W. C., Benowitz N. L., Curry S. J. et al. Chapter 3. Clinical interventions for tobacco use and dependence. In: Clinical Practice Guideline. Treating Tobacco Use and Dependence: 2008 Update. Rockville, MD: US Department of Health and Human Services. Public Health Service; 2008, pp. 37-60.

36. Miller W. R., Yahne C. E., Moyers T. B., Martinez J., Pirritano M. A randomized trial of methods to help clinicians learn motivational interviewing. J Consult Clin Psychol 2004; 72: 1050-62.

37. Mitcheson L., Bhavsar K., McCambridge J. Randomized trial of training and supervision in motivational interviewing with adolescent drug treatment practitioners. J Subst Abuse Treat 2009; 37: 73-8.

38. West R., Hajek P., Stead L., Stapleton J. Outcome criteria in smoking cessation trials: proposal for a common standard. Addiction 2005; 100: 299-303.

39. Hughes J. R., Keely J. P., Niaura R. S., Ossip-Klein D. J., Richmond R. L., Swan G. E. Measures of abstinence in clinical trials: issues and recommendations. Nicotine Tob Res 2003; 5: 13-25.

40. Jarvis M. J., Tunstall-Pedoe H., Feyerabend C., Vesey C., Saloojee Y. Comparison of tests used to distinguish smokers from nonsmokers. Am J Public Health 1987; 77: 14358.

41. Waage H., Silsand T., Urdal P., Langard S. Discrimination of smoking status by thiocyanate and cotinine in serum, and carbon monoxide in expired air. Int J Epidemiol 1992; 21: 488-93.

42. Global Adult Tobacco Survey Collaborative Group. Global Adult Tobacco Survey (GATS): Core Questionnaire with Optional Questions, Version 2.0. Atlanta, GA: Centers for Disease Control and Prevention; 2010. Available at: http://www.who.int/tobacco/surveillance/en_tfi_gats _corequestionnairewithoptionalquestions_v2_FINAL _03Nov2010.pdf (accessed 10 July 2014) (Archived at http://www.webcitation.org/6QxXUokeg on 10 July 2014).

43. de Leon J., Diaz F. J., Becona E., Gurpegui M., Jurado D., Gonzalez-Pinto A. Exploring brief measures of nicotine dependence for epidemiological surveys. Addict Behav 2003; 28: 1481-6.

44. Mahoney J., Drinka T. J., Abler R., Gunter-Hunt G., Matthews C., Gravenstein S. et al. Screening for depression: single question versus GDS. J Am Geriatr Soc 1994; 42: 1006-8.

45. Cohen S., Kamarck T., Mermelstein R. A global measure of perceived stress. J Health Soc Behav 1983; 24: 385-96.

46. Sherbourne C. D., Stewart A. L. The MOS social support survey. Soc Sci Med 1991; 32: 705-14.

47. Ewing J. A. Detecting alcoholism. The CAGE questionnaire. JAMA 1984; 252: 1905-7.

48. Velicer W. F., Diclemente C. C., Rossi J. S., Prochaska J. O. Relapse situations and self-efficacy: an integrative model. Addict Behav 1990; 15: 271-83.

49. Prochaska J. O., DiClemente C. C. Stages and processes of self-change of smoking: toward an integrative model of change. J Consult Clin Psychol 1983; 51: 390-5.

50. Stata Corporation. Stata Statistical Software: Release 12. College Station, TX: StatCorp; 2011.

51. Louwagie G. M., Ayo-Yusuf O. A. Tobacco use patterns in tuberculosis patients with high rates of human immunodeficiency virus co-infection in South Africa. BMC Public Health 2013; 13: 1031. doi: 10.1186/1471-2458-131031.

52. Dewing S., Mathews C., Schaay N., Cloete A., Louw J., Simbayi L. 'It's important to take your medication everyday 
okay?' An evaluation of counselling by lay counsellors for ARV adherence support in the Western Cape, South Africa. AIDS Behav 2013; 17: 203-12.

53. Peltzer K., Naidoo P., Louw J., Matseke G., Zuma K., McHunu G. et al. Screening and brief interventions for hazardous and harmful alcohol use among patients with active tuberculosis attending primary public care clinics in South Africa: results from a cluster randomized controlled trial. BMC Public Health 2013; 13: 699.

54. Fairall L. R., Bachmann M. O., Louwagie G. M., van Vuuren C., Chikobvu P., Steyn D. et al. Effectiveness of antiretroviral treatment in a South African program: a cohort study. Arch Intern Med 2008; 168: 86-93.

55. Dewing S., Mathews C., Cloete A., Schaay N., Shah M., Simbayi L. et al. From research to practice: lay adherence counsellors' fidelity to an evidence-based intervention for promoting adherence to antiretroviral treatment in the Western cape, South Africa. AIDS Behav 2013; 17: 293545.

56. Landaw S. A. The effects of cigarette smoking on total body burden and excretion rates of carbon monoxide. J Occup Med 1973; 15: 231-5.

57. Ahluwalia J. S., Okuyemi K., Nollen N., Choi W. S., Kaur H., Pulvers K. et al. The effects of nicotine gum and counseling among African American light smokers: a $2 \times 2$ factorial design. Addiction 2006; 101: 883-91.

58. Faseru B., Nollen N. L., Mayo M. S., Krebill R., Choi W. S. Benowitz N. L. et al. Predictors of cessation in African American light smokers enrolled in a bupropion clinical trial. Addict Behav 2013; 38: 1796-803.

59. Lando H. A. Lay facilitators as effective smoking cessation counselors. Addict Behav 1987; 12: 69-72.
60. Andrews J. O., Felton G., Ellen Wewers M., Waller J., Tingen M. The effect of a multi-component smoking cessation intervention in African American women residing in public housing. Res Nurs Health 2007; 30: 45-60.

61. Hood N. E., Ferketich A. K., Paskett E. D., Wewers M. E. Treatment adherence in a lay health adviser intervention to treat tobacco dependence. Health Educ Res 2013; 28: $72-82$.

62. Louwagie G., Girdler-Brown B., Odendaal R., Rossouw T., Johnson S., Van der Walt M. Missed opportunities for accessing HIV care among Tshwane tuberculosis patients under different models of care. Int J Tuberc Lung Dis 2012; 16: 1052-8.

63. Department of Health Republic of South Africa. Government Gazette Vol. 554, No.34523. National Health Act (61/2003): Policy on National Health Insurance. Pretoria: Government Printing Works; 2011. 
Supplementary material S1: intervention training guide

1. Theoretical background to Motivational Interviewing

2. Basic structure and approach to brief MI used

3. Training programme overview

4. Other training sources used 


\section{Theoretical background to Motivational Interviewing}

Motivational interviewing (MI) is defined as "a directive, client-centred counselling style for eliciting behaviour change by helping clients to explore and resolve ambivalence" (Rollnick \& Miller 1995). Rather than health care providers telling patients what to do, MI relies on patients making identifying the pros and cons of their behaviour and making decisions for themselves. This does not mean that the therapist is passive: the counselor has a clear goal and pursues systematic strategies to achieve this goal. The five guiding principles of $\mathrm{MI}$ are:

(a) expressing empathy, (b) developing discrepancy, (c) avoiding argumentation (d) rolling with resistance and (e) supporting self-efficacy (Miller \& Rollnick 1991).

Expressing empathy refers to an attitude of acceptance and understanding of the clients' feelings and viewpoints. Acceptance is not the same thing as agreement or approval of the behaviour but is essential to build a working alliance with the client who now feels freed to change. Reflective listening is fundamental to this principle. Another important aspect of empathy is that the counsellor accepts ambivalence towards change as a normal part of human behaviour and not something pathological that must be eliminated (Miller \& Rollnick 1991).

The second principle of MI is to create a discrepancy between the client's present behaviour and his/her personal broader goals (such as health, family, success or positive self-image). A critical aspect of this is that strategies of MI aim to increase intrinsic motivation. The client him/herself comes up with reasons for change, also sometimes referred to as "eliciting selfmotivational talk" (Miller \& Rollnick 1991). 
The third principle is avoiding argumentation. Argumentation about the necessity for change and forcing patients to accept labels is often counterproductive. It leads to defensiveness and counter-argumentation. Resistance from the client is a signal to change counselling strategies (Miller \& Rollnick 1991).

The fourth principle refers to "rolling with resistance". This term is used in analogy with martial arts such as judo in which an attack is not met with direct opposition, but rather the attacker's own momentum is used to good advantage. One example is that statements made by clients can be turned or reframed differently, thereby creating new perceptions or viewpoints for the client. The counselor also sometimes turns problems back to clients, thus allowing the client to find his/her own solutions (Miller \& Rollnick 1991).

The last principle is supporting self-efficacy. Self-efficacy is a construct that describes a person's perceived ability to perform a given task. Perceived self-efficacy therefore deals with the notion that a person can successfully perform a behaviour required to produce an expected outcome (Bandura 1977). According to this theory methods of treatment to change behaviour alter the strength of self-efficacy. In MI self-efficacy can be supported by an emphasis on personal responsibility. No one else can make the change. Even if the client has failed in the past, there is hope in the range of alternative approaches available. Belief in the possibility of change is also a very important motivator (Miller \& Rollnick 1991).

Table 1 outlines strategies to apply the principles of $\mathrm{MI}$ in the context of smoking cessation programmes. However, Rollnick and Miller (1995) emphasize the importance of distinguishing between the spirit of $\mathrm{MI}$ and the techniques since focussing too much on matter of technique can make one lose sight of the spirit and style that are central to the approach. 
Table 1. Motivational interviewing framework

\begin{tabular}{|c|c|}
\hline Action & Strategies for implementation \\
\hline $\begin{array}{l}\text { Express } \\
\text { empathy }\end{array}$ & $\begin{array}{l}\text { - Use open-ended questions to explore: } \\
\text { - The importance of addressing smoking or other tobacco use (e.g. "How } \\
\text { important do you think it is for you to quit smoking?" } \\
\text { - } \quad \text { Concerns and benefits of quitting (e.g. "What might happen if you quit?") } \\
\text { - Reflect words or meaning (e.g. "So you think smoking helps you to maintain } \\
\text { your weight?") } \\
\text { - Summarize (e.g. "What I have heard so far is that smoking is something you } \\
\text { enjoy. On the other hand, your boyfriend hates your smoking, and you are } \\
\text { worried you might develop a serious disease".) } \\
\text { - Normalize feelings and concerns (e.g. "Many people worry about managing without } \\
\text { cigarettes".) } \\
\text { Support the patient's autonomy and right to choose or reject change (e.g. "I hear } \\
\text { you saying you are not ready to quit smoking right now. I'm here to help you when } \\
\text { you are ready".) }\end{array}$ \\
\hline $\begin{array}{l}\text { Develop } \\
\text { discrepan } \\
\text { cy }\end{array}$ & $\begin{array}{l}\text { - Highlight the discrepancy between the patient's present behaviour and expressed } \\
\text { priorities, values and goals (e.g. "It sounds like you are very devoted to your family. } \\
\text { How do you think your smoking is affecting your children?") } \\
\text { - Reinforce and support "Change talk" and "commitment" language: } \\
\text { - "So, you realize how smoking is affecting your breathing and making it hard to } \\
\text { keep up with your kids". } \\
\text { - "It's great that you are going to quit when you get through this busy time at } \\
\text { - Bork". } \\
\text { - "There are effective treatments that will ease the pain of quitting, including } \\
\text { - counselling and many medication options". }\end{array}$ \\
\hline $\begin{array}{l}\text { Roll with } \\
\text { resistance }\end{array}$ & $\begin{array}{l}\text { - Back off and use reflection when the patient expresses resistance: } \\
\text { - "Sounds like you are feeling pressured about your smoking" } \\
\text { - Express empathy: } \\
\text { - "You are worried about how you would manage withdrawal symptoms". } \\
\text { - Ask permission to provide information: } \\
\text { - "Would you like to hear about some strategies that can help you address that } \\
\text { concern when you quit?" }\end{array}$ \\
\hline $\begin{array}{l}\text { Support } \\
\text { self- } \\
\text { efficacy }\end{array}$ & $\begin{array}{l}\text { - Help the patient to identify and build on past successes: } \\
\text { - } \quad \text { "So you were fairly successful the last time you tried to quit". } \\
\text { - Offer options for achievable small steps toward change: } \\
\text { - } \quad \text { Call the quitline (1-800-QUIT-NOW) for advice and information. } \\
\text { - } \quad \text { Read about quitting benefits and strategies. } \\
\text { - } \quad \text { Change smoking patterns (e.g. no smoking in the home). } \\
\text { - } \quad \text { Ask the patient to share his or her ideas about quitting strategies. }\end{array}$ \\
\hline
\end{tabular}

Source: Fiore MC, Jaén CR, Baker TB, Bailey WC, Benowitz NL, Curry SJ, et al. 2008. US Department of Health and Human Services. Public Health Service. Clinical practice Guideline. Treating Tobacco Use and Dependence: 2008 Update 
The effectiveness of $\mathrm{Ml}$ for the treatment of a variety of medical conditions was demonstrated in a meta-analysis by Rubak et al. (2005). Pooled results of the selected studies showed a significant effect for body mass index, blood alcohol concentration, systolic blood pressure and total blood cholesterol, while combined effect estimates for $\mathrm{HbA}_{1 \mathrm{c}}$ and for cigarettes per day were not significant. Psychologists and physicians obtained an effect in a higher percentage of the studies than other health care providers $(80 \%$ vs. 46\%). Two other systematic reviews focussed specifically on the effectiveness of $\mathrm{Ml}$ for smoking cessation. Although there was considerable variation in study quality and intervention effect $\mathrm{Ml}$ yielded a small but significant increase in quitting in both these reviews (Lai et al. 2010, Heckman, Egleston \& Hofmann 2010).

$\mathrm{MI}$ techniques have been adapted to shorter forms, commonly referred to as "brief Ml" for use in busy clinical settings. In the context of smoking cessation, a simple one page approach has been proposed for clinicians consisting of a quick assessment (building rapport, assessing confidence and motivation to quit), the patient identifying problems and solutions and setting of targets and follow-up visit (Rollnick, Butler \& Stott 1997). The same approach to brief MI as outlined above was adopted for this study. 


\section{Basic structure and approach to brief MI used}

The basic structure of the MI session followed the approach outlined by Rollnick, Butler and Stott (1997), consisting of a quick assessment, the patient identifying problems and solutions and identifying a target. (Table 2)

Messaging about risks were tailored to the relationship between smoking and tuberculosis. For those patients highly motivated to quit and highly confident about his/her ability to quit, the counsellor helped the patient with a quit plan. Strategies for the implementation of a quit plan entailed amongst others: "Setting a quit date", "Tell family friends and co-workers about quitting, and request understanding and support", "Anticipate challenges to the upcoming quit attempt (including nicotine withdrawal symptoms)", "Remove tobacco products from your environment" (S T A R). In addition the counsellor provided practical problem solving and skills training to enhance self-efficacy. The intervention took about 1520 minutes.

Table 2. Brief motivational interviewing intervention (Source: Rollnick, Butler and Stott 1997)

\section{Phase I: Quick assessment}

Rapport: "What sort of smoker are you? Tell me a bit about your smoking?" "You may well be a little fed up with people lecturing you about smoking. I'm not going to do that, but it would help me if I understood how you really feel about your smoking...

Motivation to quit: "If on a scale of 1 to 10,1 is not at all motivated to give up smoking and 10 is $100 \%$ motivated to give up, what number would you give yourself at the moment?'

Confidence in ability to quit: "If you were to decide to give up smoking now, how confident are you that you would succeed? If on a scale of 1 to 10, 1 means that you are not at all confident and 10 means that you are 100\% confident you could give up and remain a non smoker, what number would you give yourself now?"

\section{Phase II: Patient identifies problems and solutions}

If it is a problem, always deal with motivation first.

Motivation

Useful questions:

- "Why are you at (chosen number) and not at 1?" 
- "What would need to happen for you to get from (chosen number) to (higher number)?

Useful strategies:

- Pros and cons. "What you like about smoking?" Pt responds, then ask "What you dislike about smoking?" Summarise both sides; then ask "Where does that leave you now?"

- Non judgmental information about personal risk. "Would up to date information about the risks involved help you in your decision making about smoking?"

\section{Confidence}

Useful questions:

- "Why are you at (chosen number) and not at 1?"

- "What would need to happen, for you to get from (chosen number) to (higher number)?

- "How can I help you get from (chosen number) to (higher number)? If no ideas come from patient, offer range of possibilities.

Useful strategy:

- $\quad$ Brainstorming solutions

○ Help patient select general problem area first (e.g. withdrawal, weight, social situations, mood states, stress).

- Don't immediately offer a single, simple solution.

- Encourage patient to say what could work (their past successes, experience of others, wild speculation)

- Supplement with your ideas

- Patient chooses best option

\section{Phase III: Target and follow up}

Target: Reinforce value of small gains and openness. Can patient set manageable goal?

- May relate to numbers of cigarettes smoked (not to increase, to cut down or quit)

- May relate to factors that influence smoking, such as relationships, weight, exercise

- If not ready to set any sort of target, keep communication open: "Things do change

\section{Follow up:}

... Can we agree to leave the door open on this one?"

Find out how best they think you can help them attain their target. Ideas could include follow up visits, telephone calls, advice on nicotine replacement (for those with definite signs of nicotine addiction only).

Source: Rollnick, S., Butler, C.C. \& Stott, N. 1997, "Helping smokers make decisions: the enhancement of brief intervention for general medical practice", Patient education and counseling, vol. 31, no. 3, pp. 191-203. 


\section{Training programme overview}

An overview of the training programme for the intervention is provided in Table 3.

The training started with the presentation of the theoretical foundations of the epidemiology of tobacco-related diseases, the background to the research project and the links between tobacco smoking and tuberculosis. These topics were followed by lectures and discussions on the WHO framework Convention on Tobacco control and understanding tobacco use and nicotine dependence and pharmacology.

Later on participants were given an introduction to behavioural therapies, the principles of $\mathrm{Ml}$ and rapport exercises (practising the principles of open vs. closed questions, reflections and expressing empathy).

On day two, MI principles were recapped and interactive group exercises were done on "dealing with resistance", "change talk" and "giving feedback".

Students then watched and discussed short video screenings of MI in medical settings.

Screenings were based on locally produced video-recordings by the group facilitator and experienced MI counselor, Professor AO Ayo-Yusuf and on videotapes produced by Miller \& Rollnick (1998, see heading 4 for more details. ) This session was followed by video-taped role plays by the participants: one participant took up the role of counselor while the other participant was the "patient" who was presented on beforehand with a scenario as of the type of smoker he/she represented. The video-taped role-plays were followed by immediate real-life feedback sessions that ensured that counsellors understood the principles and spirit of $\mathrm{MI}$, and were able to apply the primary skills of using open-end questions, affirmations, reflective listening and summaries consistently during patient encounters. Day three of the training was the same as day two, but then for another group of students. 
Some weeks after the initial three day training, students underwent an additional one-day training, to strengthen and deepen their counselling skills. During the actual trial roll-out, on site follow-up practical sessions were organised about every four months whereby non-videotaped role plays between the research assistant and the counsellors were performed.

Table 3. Training programme overview

Day 1 (Group 1 and 2)

Presenters: AO Ayo-Yusuf and G Louwagie

Time Item

08.15-8.30 Tea

08:30-08:45 Welcome \& introductions

08:45-09:45 Research project overview: Tobacco smoking and tuberculosis

09:45-10:15 Epidemiology of tobacco-related diseases

\section{Tea}

10:45-11:15 WHO Framework Convention on Tobacco Control (FCTC)

11:15-12:10 Understanding tobacco use/nicotine dependence - profile of a smoker and psychological factors associated with smoking.

12:10-12:40 Nicotine pharmacology

12:40-13:40 Lunch

13:40-14:10 Introduction to behavioural theories

14:10-14:45 'Turn to your neighbour' rapport exercise

14:45-15:00 Tea break

15:00-16:00 Principles of Motivational Interviewing (MI)

16:00 Adjournment 


\section{Day 2 (Group 1)}

Presenters: AO Ayo-Yusuf

Time Item

09:00-09:30 MI principles recap

09:30-11:00 Work in groups on Ml exercises: Dealing with 'resistance' and starting 'change talk' and giving 'feedback'

11:00-11:30 Video screening on $\mathrm{Ml}$ in a medical setting

11:30-11:45 Tea break

11:45-13:00 Role plays MI smoking scenarios (15 min per participant)

13:00-14:00 Lunch

14:00-15:00 Role plays MI smoking scenarios (15 min per participant) Implementation challenges and solutions

15:00 Adjournment

\section{Day 3 (Group 2)}

Presenters: AO Ayo-Yusuf

Time Item

09:00-09:30 MI principles recap

09:30-11:00 Work in groups on Ml exercises: Dealing with 'resistance' and starting 'change talk' and giving 'feedback'

11:00-11:30 Video screening on $\mathrm{Ml}$ in a medical setting

11:30-11:45 Tea break

11:45-13:00 Role plays MI smoking scenarios (15 min per participant)

13:00-14:00 Lunch

14:00-15:00 Role plays MI smoking scenarios/Implementation of challenges and solutions

15:00 Adjournment 


\section{Follow-up training (1 dav)}

Presenter: G Louwagie and AO Ayo-Yusuf

Time Item

08:30-08:45 Welcome \& introductions

08:45-09:15 Recap of Epidemiology of tobacco-related diseases/TB and smoking

09:15-09: 45 Recap of Understanding tobacco use/nicotine dependence

09.45-10.30 Motivational interviewing principles + short video screening of $\mathrm{MI}$ in medical setting

Tea

10:45-11:30 Group work: Open vs. closed questions. Reflections.

11:15-11:45 Long video screening with critical discussion

11:45-12:40 Role plays (15 minutes per participant +5 minutes discussion)

13.00-13:40 Lunch

13:45-14:45 Role plays MI smoking scenarios (15 min per participant)

14:45-15:00 Tea break

15:00-15:30 Role plays MI smoking scenarios (15 min per participant)

15:30-16.00 Operational issues and wrap-up

Adjournment 


\section{Other external sources used for the MI training}

Motivational Interviewing

Professional Training VHS Videotape/DVD Series 1998

William R. Miller, Ph.D., \& Stephen Rollnick, Ph.D.

Directed by Theresa B. Moyers, Ph.D.

The University of New Mexico

Center on Alcoholism Substance Abuse, and Addictions (UNM/CASAA) Available for

sale at: http://casaa.unm.edu/download/mitrain98.pdf (last accessed 3101 2014)

The following information regarding the contents of these CDs is provided by the authors:

"This series of six videotapes or two DVDs, produced at the University of New Mexico, is intended to be used as a resource in professional training, offering six hours of clear explanation and practical modeling of component skills. Because it is helpful to see how a method is practiced in various contexts, the tapes include clinical demonstrations of the skills of motivational interviewing, showing ten different therapists working with twelve clients who bring a variety of problems. Drs. Miller and Rollnick are deeply grateful to Terri Moyers, who volunteered many weeks of her time for the planning, directing, editing, and completion of these tapes.

A. Introduction to Motivational Interviewing. The introductory tape is a conversational interview with Bill Miller and Steve Rollnick, conducted in the summer of 1997 by Theresa Moyers. They review the background and current directions of motivational interviewing, explore its essential theoretical and conceptual underpinnings, and discuss its five basic principles. This is by no means a comprehensive introduction to motivational interviewing. Rather, it sets the context for the demonstration tapes that follow.

B. Phase 1: Opening Strategies. This is the most complex of the tapes, and spans two videocassettes. It is designed to illustrate the skills involved in the opening phase of motivational interviewing. Phase I focuses on identifying and strengthening the person's intrinsic motivation for change. It begins with the first contact and continues until the transition into Phase II, illustrated on Tape 6.

C. Handling Resistance. Motivational interviewing includes a set of strategies for handling and decreasing resistance. The information presented in this videotape is particularly useful during Phase I, although the methods are applicable throughout 
counseling. The phenomenon of "resistance" is discussed, and various strategies are explained and demonstrated.

D. Feedback and Information Exchange. One context in which motivational interviewing has been widely practiced is the "check-up" or feedback of assessment information. This specialized application involves much more talking on the part of the therapist, in that more information is being imparted to the client. How does one take this more active, information-giving role and still be consistent with the spirit of motivational interviewing? That is the focus of this tape.

E. Motivational Interviewing in Medical Settings. A rapidly growing application of motivational interviewing is in general health care settings. Here it is often necessary to compress the process of counseling into a shorter period of time. This tape explores how the spirit of motivational interviewing can be applied in busy health care settings.

F. Phase 2: Moving Toward Action. How do you know when to move from Phase 1 (building motivation for change) into Phase 2 (consolidating commitment to a change plan)? What counseling methods are used in Phase 2, and how do they differ from the opening strategies of motivational interviewing? That is the focus of the final tape in this series."

Available in VHS Tapes, DVDs, and now VHS PAL (European Format)

For additional information, please contact:

Sylvia Law

UNM/CASAA

MSC11 6280, 1 University of New Mexico

Albuquerque, NM 87131-0001 USA
Phone: 505-925-2378

Fax: 505-925-2379

Email: slaw@unm.edu 


\section{References}

Bandura, A. 1977, "Self-efficacy: toward a unifying theory of behavioral change", Psychological review, vol. 84, no. 2, pp. 191-215.

Fiore MC, Jaén CR, Baker TB, Bailey WC, Benowitz NL, Curry SJ, et al. 2008, Clinical Practice Guideline. Treating Tobacco Use and Dependence: 2008 Update. US Department of Health and Human Services. Public Health Service, Rockville, Maryland.

Heckman, C.J., Egleston, B.L. \& Hofmann, M.T. 2010, "Efficacy of motivational interviewing for smoking cessation: a systematic review and meta-analysis", Tobacco control, vol. 19, no. 5, pp. 410-416.

Lai, D.T., Cahill, K., Qin, Y. \& Tang, J.L. 2010, "Motivational interviewing for smoking cessation", Cochrane database of systematic reviews (Online), vol. (1), no. 1, pp. CD006936.

Miller WR, Rollnick.S. 1991, " Chapter 5. Principles of Motivational Interviewing" in Motivational interviewing. Preparing People to Change Addictive Behaviours. The Guilford Press, New York/London, pp.51-63.

Rollnick S, M.W. 1995, "What is motivational interviewing?", Behavioral and Cognitive Psychotherapy, vol. 23, pp. 325-334.

Rollnick, S., Butler, C.C. \& Stott, N. 1997, "Helping smokers make decisions: the enhancement of brief intervention for general medical practice", Patient education and counseling, vol. 31, no. 3, pp. 191-203. 